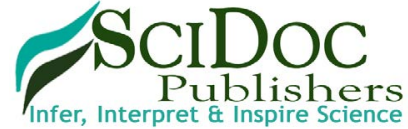

International Journal of Surgery and Research (IJSR)

ISSN 2379-156X

\title{
The Use of Point of Care Testing for Monitoring of APTT in Patients Receiving Heparin Infusion
}

\author{
Research Article
}

Surman TL*, Tran M, Worthington $\mathrm{MG}^{*}$

D’arcy Sutherland Cardiothoracic Surgery Unit, Royal Adelaide Hospital, Adelaide, South Australia.

\section{Abstract}

Background: There is a high risk of thromboembolic events among patients undergoing cardiac surgery, and perioperative anticoagulation is sometimes required. The time taken for laboratory based APTT blood sampling results and adjustments may compromise patient safety. There is evidence in the literature to suggest that bedside testing turnaround times is of benefit to inpatient care, and conversely that POCT is inaccurate. The primary aim of this study was to determine if point of care testing of APTT was accurate when compared to laboratory based APTT measurements. The secondary aim was to identify if there was a correlation between operator experience and accuracy of results.

Methods: Following full ethics approval by the Royal Adelaide Hospital, Human Research Ethics Committee (HREC), current Cardiothoracic Surgery Unit inpatients were enrolled in the study over a period from July 2016-March 2017. A total of 50 samples were obtained from the minimum number of patients required and separated into three tiers; point of care testing group from finger prick test, point of care testing group from standard postoperative blood sample, and laboratory based testing of standard postoperative blood sample. Results were correlated with Central Adelaide Local Health Network (CALHN) heparin infusion protocols. Clinically significant samples were those which POCT IV and laboratory IV results varied enough to alter the heparin infusion protocol rate change.

Results: Demographics showed $83 \%$ of patients were male, $67 \%$ were receiving IV heparin preoperatively for unstable coronary artery disease, and the mean age of participants was 66 years. Of the 50 collected samples, $17 / 50(34 \%)$ were recorded as being clinically significant. On the adjusted POCT protocol, $14 / 50(28 \%)$ of samples were clinically significant. There was a statistically significant difference between POCT IV and laboratory IV results; $p=0.02$ and mean difference between values was 19.1. Linear regression analysis showed a poor correlation between POCT IV and laboratory IV samples. There was no relationship between preoperative and postoperative samples.

Conclusions: Point of care testing in our study of 50 patient samples shows that there is a poor correlation between POCT and laboratory based testing. Additionally, there is a steep learning curve for those performing the test with no marked improvement following progressive samples. Accordingly, POCT testing in cardiac surgery patients is not recommended for clinical use until further research is completed showing improved correlation between tests, and methods to overcome the steep learning curve are identified.

Abbreviations: APTT: Activated Partial Thromboplastin Time; POCT: Point-of-Care Testing; HREC: Human Research Ethics Committee; CALHN: Central Adelaide Local Health Network; UFH: Unfractionated Heparin.

\section{Background}

Patients on intravenous heparin require regular Activated Partial Thromboplastin Time (APTT) monitoring. It has been suggested in the literature that while POC testing devices provide rapid results, such devices may sacrifice accuracy and precision. Conflicting reports do exist in the literature and discuss either a poor correlation between centralized laboratory tests and POCT for the APTT, or that the POCT testing for UFH may be affected by other drugs, or that correlation of APTT with heparin concentration may vary with the APTT instrument [1-16]. The aim of this study is to determine whether APT'T Point of Care Testing (POCT) in post-operative cardiac surgery patients on heparin infusion is accurate when compared to

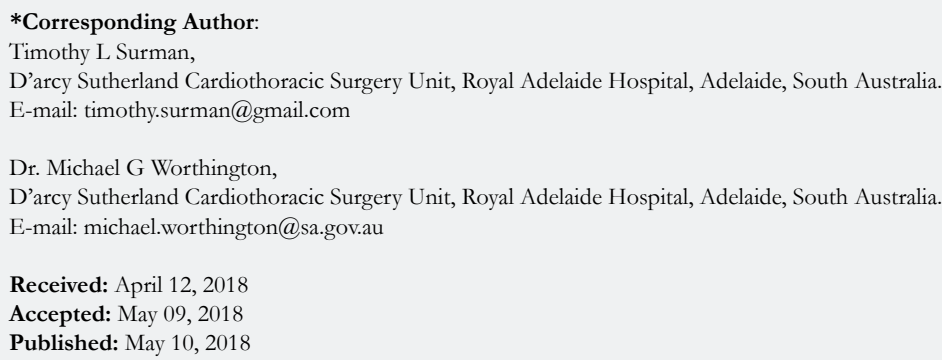

Citation: Surman TL, Tran M, Worthington MG. The Use of Point of Care Testing for Monitoring of APTT in Patients Receiving Heparin Infusion. Int J Surg Res. 2018;5(3):100-106. doi: http://dx.doi.org/10.19070/2379-156X-1800022

Copyright: Surman TL $^{\circ}$ 2018. This is an open-access article distributed under the terms of the Creative Commons Attribution License, which permits unrestricted use, distribution and reproduction in any medium, provided the original author and source are credited. 
standard laboratory based monitoring, and whether any reported inaccuracies correlate clinically with standardised heparin infusion management protocols.

\section{Methods}

Following full ethical approval by the Royal Adelaide Hospital Human Research Ethics Committee, cardiac surgery patients undergoing intravenous heparin infusion for perioperative anticoagulation were enrolled in our study over a period from July 2016 to March 2017.

The inclusion criteria were patients who were receiving preoperative or postoperative heparin infusion therapy for high risk coronary disease or bridging therapy from or to warfarin. Exclusion criteria were known malignancy, patients with a known coagulation disorder or patients recently receiving NOAC therapy. As this study would involve additional finger prick and venepuncture in addition to standard lab testing, patients who agreed participated in providing samples on multiple occasions to ensure the minimum patients required were utilised to achieve the study aims. In order to minimise the amount of physical venepuncture on multiple patients, the focus was to collect 50 total samples using the least amount of patients possible to reach this sample goal. Selected patients were asked to participate following an explanation of the study protocol and appropriate consent. The Hemochron Signature Elite Jr point of care testing device (Zoll Medical, Australia) with APTT compatible cuvettes was utilised for testing.

Blood was collected using a $10 \mathrm{ml}$ syringe in an $18 \mathrm{~g}$ needle in the cubital fossa from all patients. The blood from the $10 \mathrm{ml}$ syringe was transferred to a blue citrate tube, and to the POCT cuvette within 10 seconds for all patients. Immediately following this, a finger prick was performed using a dedicated finger prick needle and the blood inserted directly into the POCT cuvette within 10 seconds of the initial finger prick.

The POCT testing process involves the use of a hemochron junior portable machine and display and a hemochron junior APTT whole blood cuvette which stores the blood sample. The reagent used in the APT'T cuvettes are a silicate substance called kaolin. Performing a patient test involves the following:

1. Hemochron device is turned on and refrigerator stored cuvette is inserted into the machine when at room temperature. The cuvette then undergoes a warming up period before testing is allowed.
2. Blood sample is taken via intravenous or finger prick sample. 3. One drop of blood is dispensed onto the cuvette into the cuvette sample well with no dips or wells allowed in the sample well.

4. 15 micro/litres of blood then flow down testing channel (with excess blood flowing into the waste channel).

5. Blood then moves back and forth in the restriction well until a clot is formed and an APT'T time is displayed on the testing machine. This is an APTT whole blood value (Figure 1).

The results of these three groups are compared and analysed by identifying the APTT whole blood time in POCT and laboratory groups and the time taken to obtain these results from the POCT device and formal laboratory results.

Collected blood in citrate tubes were sent to SA Pathology at the Royal Adelaide Hospital. Following consultation with the haematology laboratory scientist; blood was analysed using 2 specific reagents.

\section{Apt thcl (acyl protein thioesterase) \\ 2. Triniclot-aptt s}

The APTT sample was then processed in the Sta-R max haemostasis analyser (Diagnostica Stago, S.A.S. France). Testing time was dependent on multiple factors including waiting time for centrifuge and testing time was prolonged in patients on heparin infusion. If samples were collected from the ward (as in our study) then times for delivery to the lab were also prolonged. Fifty samples were taken comprising preoperative and postoperative patients, and 24 patients were involved in the study. The APT'T results were calibrated against the standardised heparin infusion protocol used by The Royal Adelaide Hospital (Figure 2) to determine if the variation between POCT and laboratory testing inferred any change in heparin infusion rate and was clinically significant. Using the original protocol range as a guide, we extended the APT'T ranges to allow for greater variation with POCT. We wanted to see if a greater APTT range would allow for more consistent outcomes between Laboratory results and POCT and consistent changes in heparin infusion rates. No POCT data was used clinically in the adjustment of IV heparin infusion rates. Standard laboratory samples were used for this purpose.

The D'Agostino and Pearson omnibus normality test was used to test for normality in all cohorts. The Mann-Whitney U test was used to determine if there was a significant difference between cohorts. A linear regression analysis was performed to determine if there was a correlation between cohorts. A graph was created

Figure 1. Hemochron cuvette with labelled channels for placement of blood during POCT.

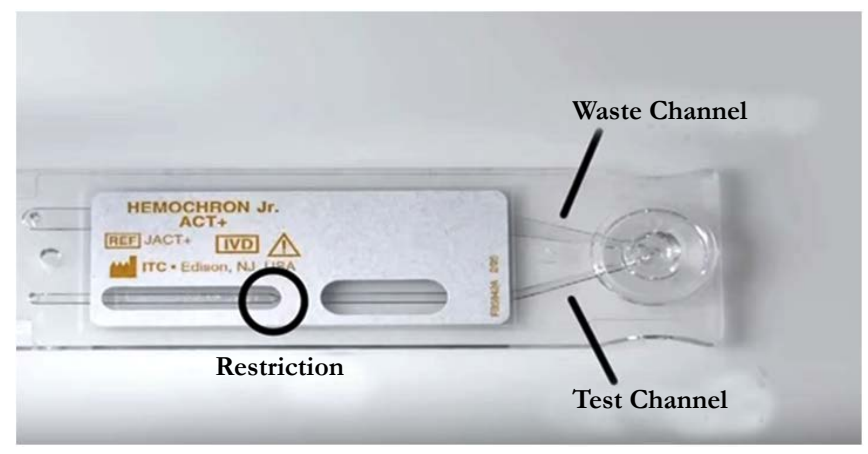


that showed the difference between IV Lab test results and POCT IV test results in order of recruitment to the study in order to observe if there was a learning curve associated with using the POCT machine.

\section{Results}

Twenty four patients agreed to participate in the study. Twenty out of $24(83 \%)$ were male, and 4/24 (16\%) were female. Mean age was 66 years $n=24$, and age range was $46-84$ years $n=24$. The indication for use of IV heparin was unstable coronary artery disease in 16/24 patients (67\%), and bridging heparin therapy in $8 / 24$ patients $(33 \%)$.

Testing times were much shorter in POCT (results available in minutes compared to up to 1 hour or more). The mean testing time for POCT IV was 110.02 (sec) $\mathrm{n}=50$, POCT FP was $111.06(\mathrm{sec}) \mathrm{n}=50$, and for IV was 98.56 (minutes) $\mathrm{n}=50$. Of the 50 samples, 1 in 3 results (34\%) showed a clinically significant variation between POCT and Lab APT'T results which would influence heparin infusion rates. The mean difference between POCT IV and Lab IV testing was 24.93 (sec). With an adjusted APT'T protocol that allowed for greater range between POCT APTT times, mean POCT IV APTT was 68.98 (sec) $n=50$, mean IV APTT times was 69.28 (sec) $n=50$, with mean difference being 0.30 (sec). Fourteen of fifty samples $(28 \%)$ were recorded as being clinically significant or showing enough variation to be clinically significant despite the adjusted infusion table parameters. If two samples (sample 10 and 35) are removed due to very high out of proportion test results then $12 / 50(24 \%)$ are clinically significant. This shows a $6 \%$ reduction in clinically significant samples when using the adjusted POCT protocol which includes a much wider POCT APTT range.

There was a statistically significant difference in the median of POCT IV vs. Lab IV, p $=0.02$. The median POCT IV APTT was 55.90 (range 34-397). The median Lab IV APT'T was 75 (range: 28 - 129). There was no difference in POCT IV vs. POCT FP (finger-prick testing), $\mathrm{p}=0.98$. Median 55.90 (range 34-397.7) versus 55.20 (36-344) respectively. The median across all tests were not statistically different using Man Whitney $U$ test $\mathrm{p}>0.05$.

Linear regression analysis showed a poor correlation between POCT (IV or FP) and Lab IV testing, correlation co-efficient $=$ 0.4 (Figure 3-7). The POCT device had good precision and FP and IV samples were comparable. The methods and techniques involved in point of care testing remained very sensitive and were associated with a significant learning curve. When results of each sample were taken it was noted that with each attempt there was some progressive improvement in accuracy between POCT and IV sampling (Figure 8). However, this improvement available varies in the laboratory analysis in both storage, processing and calibration and therefore there is no current good option for POCT in heparin infusion monitoring and adjustment at this time.

Figure 2. Heparin infusion protocol guideline used in the Royal Adelaide Hospital, Adelaide, South Australia.

Heparin Infusion Protocol

Only to be used for APTT samples sent to IMVS in patients with normal baseline tests

PERFORM BASELINE TESTS (AP'T'T, platelet count, INR)

IF ANY RESULTS IS ABNORMAL, CONTACT HAEMATOLOGY

STARTING DOSE:

\begin{tabular}{|c|c|c|c|c|c|c|}
\hline Patient weight & $\mathbf{4 6 - 5 5} \mathbf{~ k g}$ & $\mathbf{5 6 - 6 5} \mathbf{~ k g}$ & $\mathbf{6 6 - 7 5} \mathbf{~ k g}$ & $\mathbf{7 6 - 8 5} \mathbf{~ k g}$ & $\mathbf{8 6 - 9 5} \mathbf{~ k g}$ & $\mathbf{> 9 5} \mathbf{~ k g}$ \\
\hline $\begin{array}{c}\text { Administer IV bolus (units) use } \\
5,000 \text { units/5mL ampoule }\end{array}$ & 3,500 & 4,200 & 4,900 & 5,600 & 6,300 & 7,000 \\
\hline $\begin{array}{c}\text { And also begin IV infusion } \\
\text { infusion rate (units/h) } \\
\text { use 25,000 units/50mL syringe }\end{array}$ & 900 & 1,100 & 1,250 & 1,400 & 1,600 & 1,800 \\
\hline
\end{tabular}

Check APTT 6 Hours commencing heparin.

Mark all APTT request forms and blood tubes with "urgent - on heparin" sticker.

INFUSION ADJUSTMENT TABLE:

\begin{tabular}{|c|c|c|c|c|}
\hline APTT (seconds) & IV BOLUS & STOP INFUSION & RATE CHANGE & REPEAT APTT \\
\hline$<37$ & 5,000 units & & increase by 400 units/hour & 6 hours \\
\hline $38-64$ & & & increase by 200 units/hour & 6 hours \\
\hline $65-110$ & & & no change & $\begin{array}{c}\text { Daily -if stable } \\
\text { otherwise } 6 \text { hours }\end{array}$ \\
\hline $111-130$ & & & decrease by 50 units/hour & 6 hours \\
\hline $131-140$ & & 30 minutes & decrease by 100 units/hour & 6 hours \\
\hline $141-150$ & & 60 minutes & decrease by 150 units/hour & 6 hours \\
\hline$>150$ & & $\begin{array}{c}120 \text { minutes, do not restart } \\
\text { until APTT }<150\end{array}$ & $\begin{array}{l}\text { decrease by } 200 \text { units/hour } \\
\text { when restarting }\end{array}$ & 2 hours \\
\hline
\end{tabular}


Table 1. Demographics for patient's enrolled in APTT study.

\begin{tabular}{|c|c|c|}
\hline Age & Sex & Reason for IV heparin \\
\hline 68 & Male & unstable CAD \\
\hline 46 & Male & unstable CAD \\
\hline 60 & Female & unstable CAD \\
\hline 55 & Male & unstable CAD \\
\hline 81 & Male & unstable CAD \\
\hline 61 & Female & unstable CAD \\
\hline 62 & Male & unstable CAD \\
\hline 60 & Female & unstable CAD \\
\hline 78 & Male & unstable CAD \\
\hline 46 & Male & unstable CAD \\
\hline 81 & Male & unstable CAD \\
\hline 65 & Male & unstable CAD \\
\hline 77 & Male & unstable CAD \\
\hline 71 & Male & unstable CAD \\
\hline 72 & Female & Bridging to warfarin \\
\hline 55 & Male & Bridging to warfarin \\
\hline 81 & Male & Bridging to warfarin \\
\hline 52 & Male & Bridging to warfarin \\
\hline 58 & Male & Bridging to warfarin \\
\hline 79 & Male & Bridging to warfarin \\
\hline 71 & Male & Bridging to warfarin \\
\hline 84 & Male & unstable CAD \\
\hline 70 & Male & unstable CAD \\
\hline 49 & Male & Bridging to warfarin \\
\hline
\end{tabular}

Table 2. Statistical significance and normality testing across all modalities.

\begin{tabular}{|c|c|c|c|}
\hline Statistical result & POCT versus Lab & POCT IV versus POCT FP & POCT FP versus Lab IV \\
\hline Mann Whitney U Test & & & \\
\hline P value & 0.1256 & 0.9397 & No \\
\hline $\begin{array}{c}\text { Significant difference } \\
\mathrm{p}<0.05\end{array}$ & No & $\boldsymbol{P O C T} \boldsymbol{F P}$ & Lab $\boldsymbol{I V}$ \\
\hline & $\boldsymbol{P O C T \boldsymbol { V }}$ & & \\
\hline $\begin{array}{c}\text { D'Agostino and Pearson } \\
\text { omnibus normality Test }\end{array}$ & & $<0.0001$ & 0.1343 \\
\hline P value & $<0.0001$ & No & Yes \\
\hline Passed normality & No & & \\
\hline
\end{tabular}

Laboratory AP'T testing therefore remains the standard of care. For the future monitoring and adjustment of APT'T POC, tests are being developed for the $\mathrm{Xa}$ inhibitors that may be able to adapt for heparin. As the goal of this study was a clinical focus to determine if there was a clinical significant difference between POCT and Lab based testing, the gold standard of testing was Lab APTT results, instead of a plasma heparin level because this is not used routinely to monitor and adjust heparin infusion rates in our institution. As demonstrated in the (Table 2) and (Figure 7), this suggests that there is an inherent inaccuracy in the POCT process that is not attributable to operator experience.

\section{Conclusions}

There is evidence in the literature to suggest that bedside testing turnaround times is of benefit to inpatient care, however there is also evidence to suggest POCT is inaccurate. Our data has shown that point of care testing using finger prick sampling and intravenous blood testing is not comparable with laboratory based measurements and has an early learning curve that is difficult to deploy amongst a high turnover of staff members. Our data has also interestingly shown that with a modified heparin infusion protocol to allow for increased or decreased maximum or minimum range values for POCT, correlation between POCT and laboratory testing did not improve significantly. This further 
Figure 3. Analysis showed a poor correlation between all three tiers of testing.

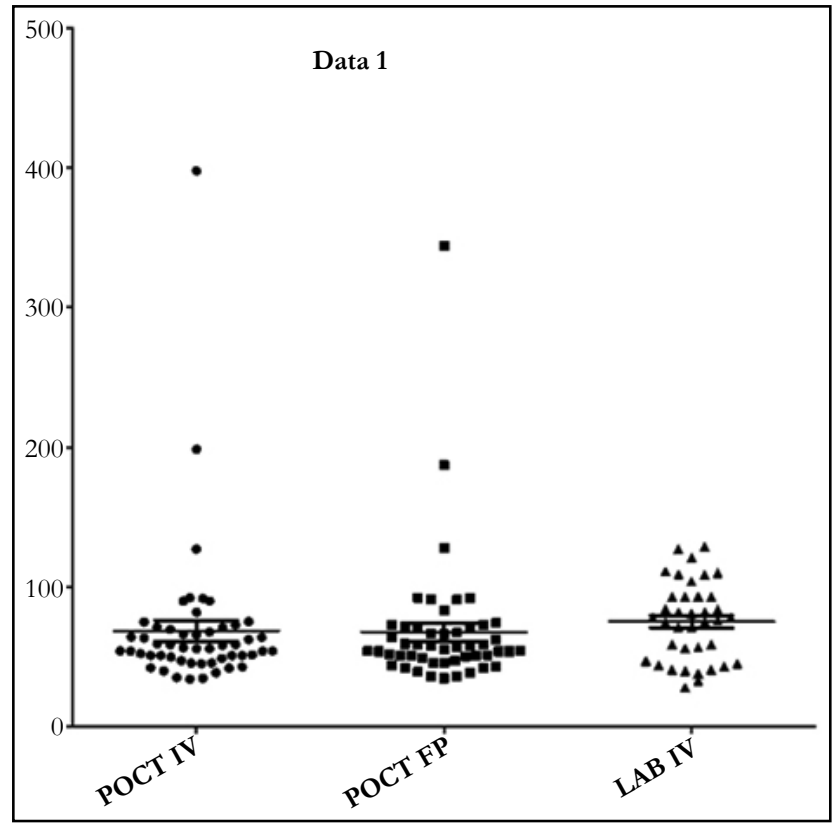

Figure 4. Analysis showed a poor correlation in comparison between the tiers of testing except POCT FP versus POCT IV which showed a strong correlation.

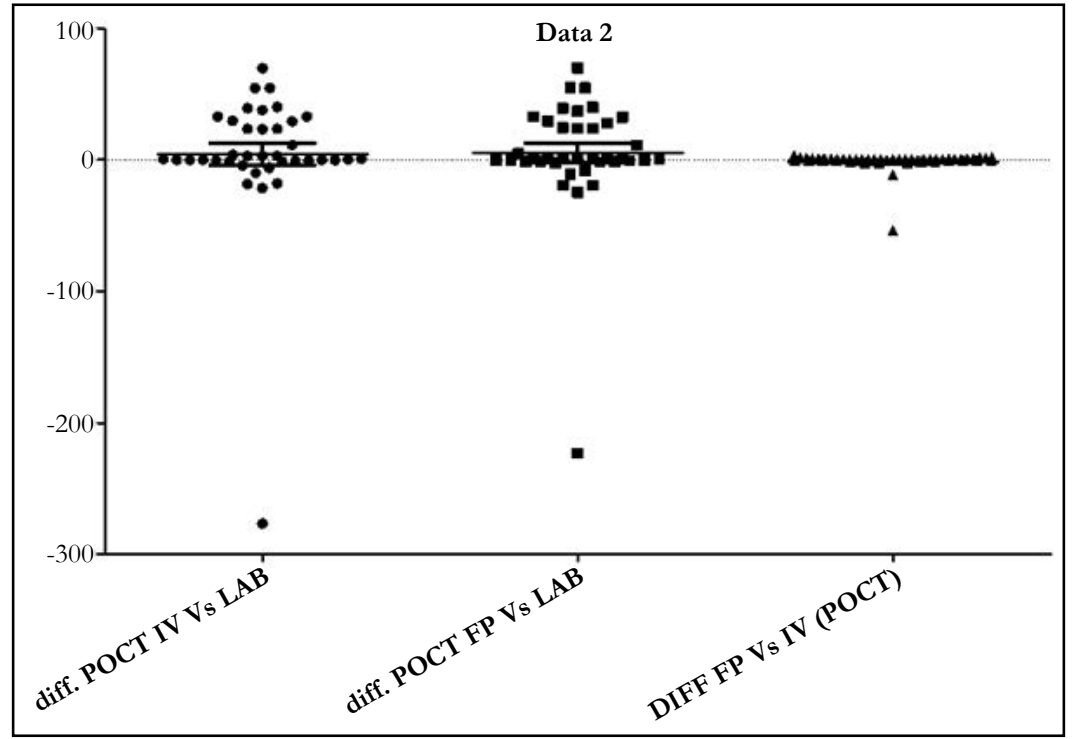

Figure 5. POCT IV versus LAB IV showing a range of data measurements on linear regression analysis.

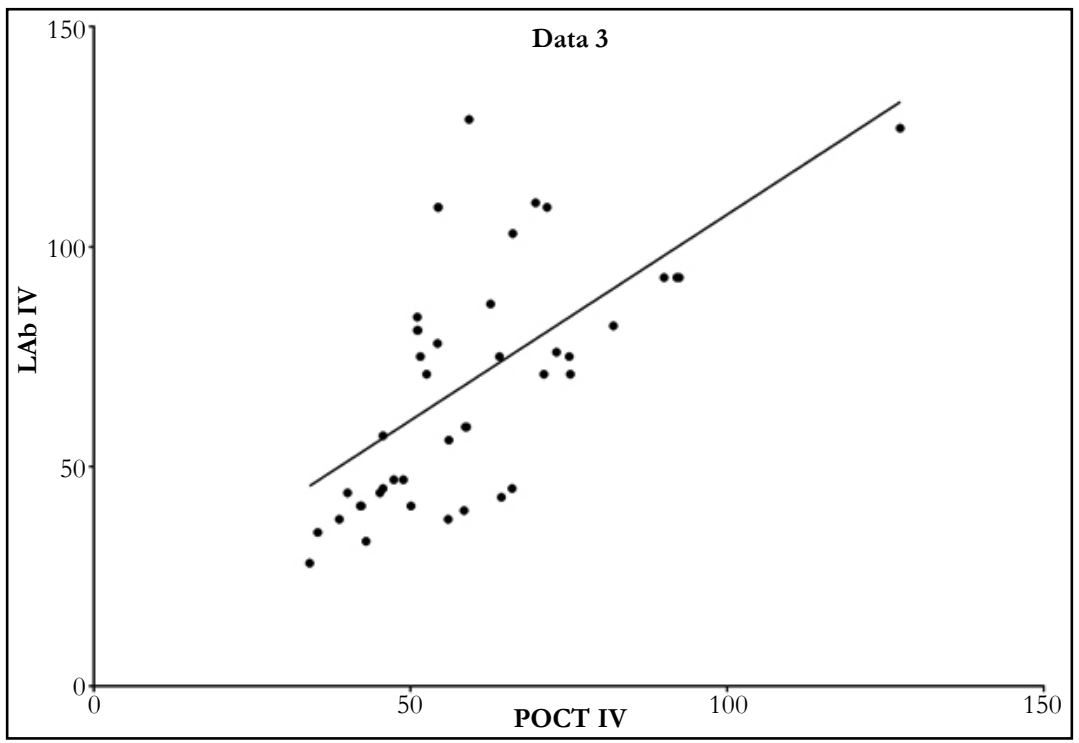


Figure 6. POCT FP versus LAB IV showing a range of data measurements on linear regression analysis.

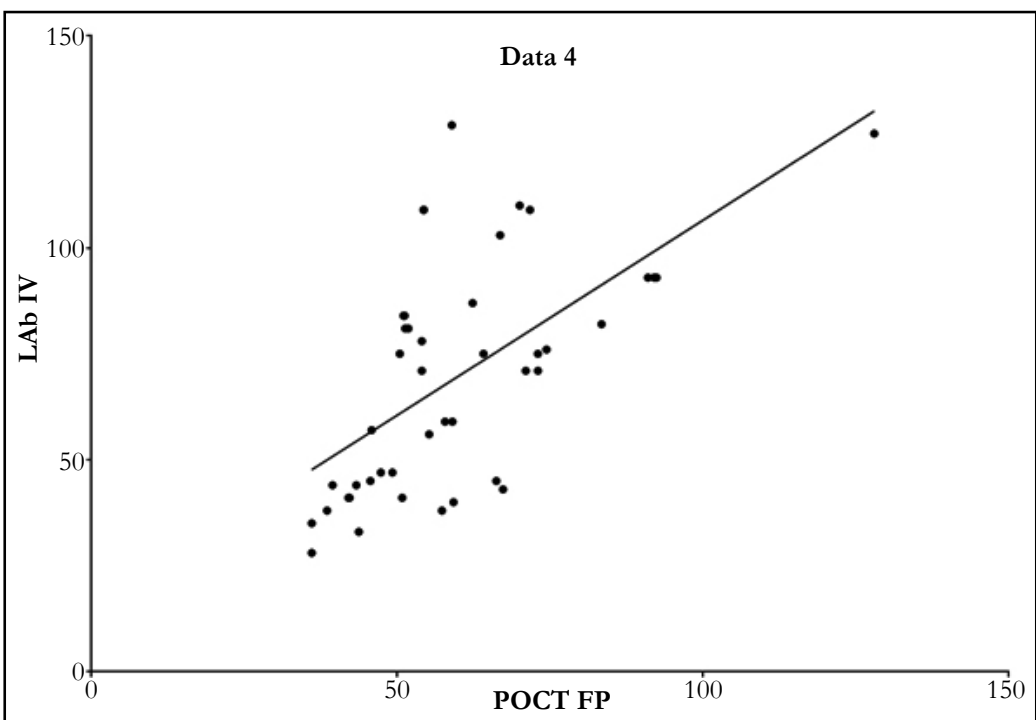

Figure 7. POCT IV versus POCT FP showing a clear linear relationship between the two test modalities.

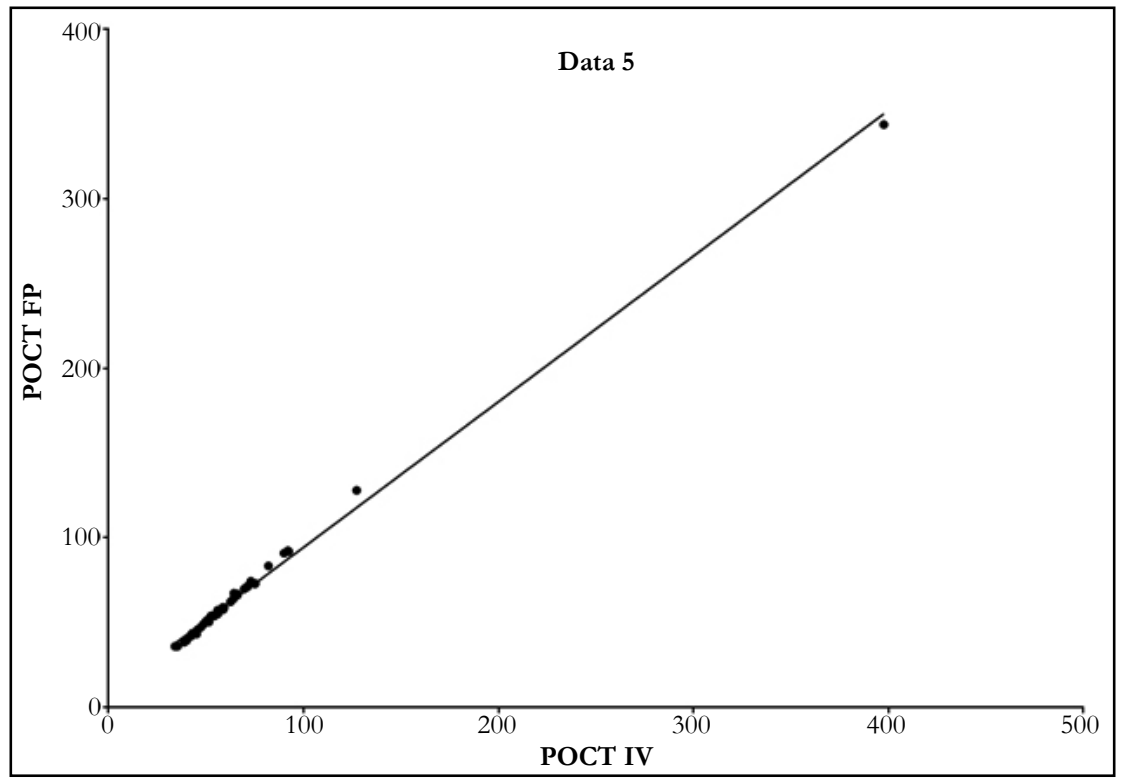

Figure 8. Learning curve graphic results.

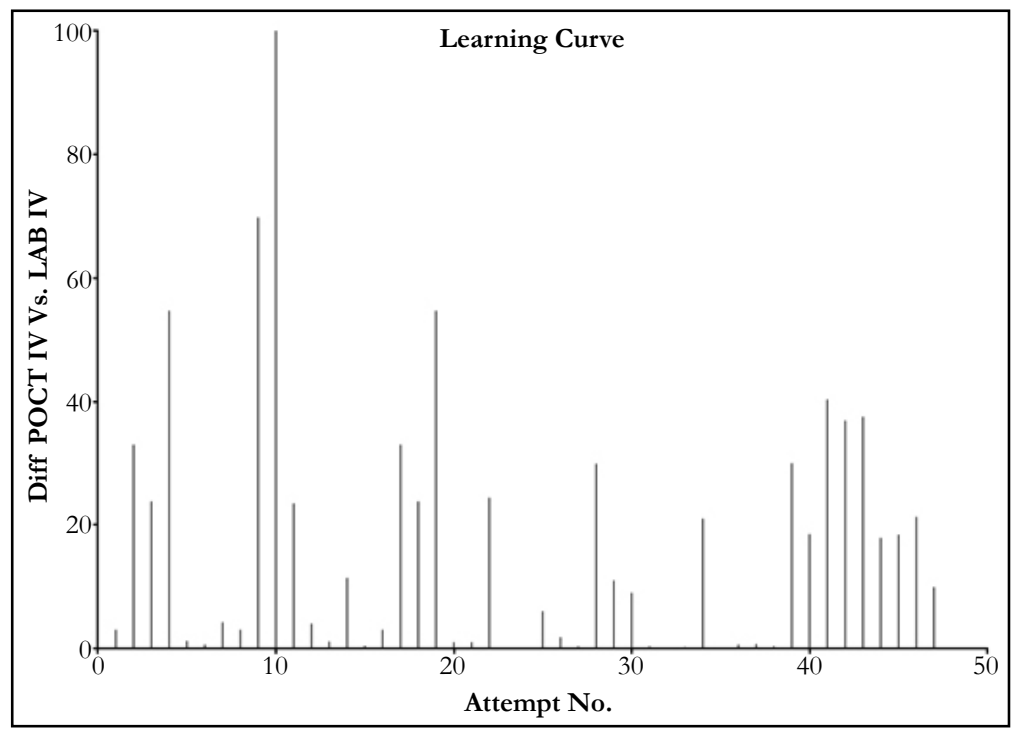


supports that testing methods are not comparable in this sample size and clinical use of POCT devices for heparin infusion adjustment would not be recommended, and laboratory based testing remains the standard of care.

\section{References}

[1]. Dager WE, Gosselin RC, Raschke CR, Vanderveen T. Heparin: Improving Treatment and Reducing Risk of Harm. Clin Lab and Saf Chal. 2009 Jan.

[2]. Perry DJ, Fitzmaurice DA, Kitchen S, Mackie IJ, Mallett S. Point-of-care testing in haemostasis. Br J Haematol. 2010 Sep;150(5):501-14. PubMed PMID: 20618331.

[3]. Ganter MT, Hofer CK. Coagulation monitoring: current techniques and clinical use of viscoelastic point-of-care coagulation devices. Anesth Analg. 2008 May;106(5):1366-75. PubMed PMID: 18420846.

[4]. Prisco D, Paniccia R. Point-of-care testing of hemostasis in cardiac surgery. Thromb J. 2003 May 6;1(1):1. PubMed PMID: 12904262

[5]. Murphy GJ, Reeves BC, Rogers CA, et al. Increased mortality, postoperative morbidity, and cost after red blood cell transfusion in patients having cardiac surgery. Circulation. 2007 Nov 27;116(22):2544-52. PubMed PMID: 17998460

[6]. Weber CF, Görlinger K, Meininger D, Herrmann E, et al. Point-of-Care TestingA Prospective, Randomized Clinical Trial of Efficacy in Coagulopathic Cardiac Surgery Patients. Anesthesiology. 2012 Sep;117(3):531-47. PubMed PMID: 22914710.

[7]. Avidan MS, Alcock EL, Da Fonseca J, Ponte J, et al. Comparison of structured use of routine laboratory tests or near-patient assessment with clinical judgement in the management of bleeding after cardiac surgery. $\mathrm{Br} \mathrm{J}$ Anaesth. 2004 Feb;92(2):178-86. PubMed PMID: 14722166.

[8]. Kulik A, Rubens FD, Wells PS, Kearon C, et al. Early postoperative anti- coagulation after mechanical valve replacement: a systematic review. Ann Thorac Surg. 2006 Feb;81(2):770-81. PubMed PMID: 16427905.

[9]. Olson J, Arkin C, Brandt J, Cunningham M, et al. College of American Pathologists Conference XXXI on laboratory monitoring of anticoagulant therapy: laboratory monitoring of unfractionated heparin therapy. Arch Pathol Lab Med. 1998 Sep;122(9):782-98. PubMed PMID: 9740136.

[10]. Ojito JW, Hannan RL, Burgos MM, et al. Comparison of point-of-care activated clotting time systems utilized in a single pediatric institution. J Extra Corpor Technol. 2012 Mar;44(1):15-20. PubMed PMID: 22730859.

[11]. Ganter MT, Hofer CK. Coagulation monitoring: current techniques and clinical use of viscoelastic point-of-care coagulation devices. Anesth Analg. 2008 May;106(5):1366-75. PubMed PMID: 18420846.

[12]. Douglas AD, Jefferis J, Sharma R, Parker R, Handa A, Chantler J. Evaluation of point-of-care activated partial thromboplastin time testing by comparison to laboratory-based assay for control of intravenous heparin Angiology. 2009 Jun-Jul;60(3):358-61. PubMed PMID: 19398428.

[13]. Choi TS, Greilich PE, Shi C, Wilson JS, Keller A, Kroll MH. Point-of-care testing for prothrombin time, but not activated partial thromboplastin time, correlates with laboratory methods in patients receiving aprotinin or epsilonaminocaproic acid while undergoing cardiac surgery. Am J Clin Pathol. 2002 Jan;117(1):74-8. PubMed PMID: 11789734.

[14]. Paniccia R, Fedi S, Carbonetto F, Noferi D, et al. Evaluation of a New Pointof-care Celite-activated Clotting Time Analyzer in Different Clinical SettingsThe i-STAT Celite-activated Clotting Time Test. Anesthesiology. 2003 Jul;99(1):54-9. PubMed PMID: 12826842.

[15]. B Nasrallah F, Graham S, Lajara RB. Comparison of five point-of-care prothrombin and activated partial thromboplastin time devices based on age of blood sample. J Extra Corpor Technol. 2002 Sep;34(3):178-81. PubMed PMID: 12395962.

[16]. Hirsch J, Wendt T, Kuhly P, Schaffartzik W. Point-of-care testing. Anaesthesia. 2001 Aug 1;56(8):760-3. 